\title{
TRANSPORTE FERROVIARIO Y PRIVATIZACIÓN
}

Jaime R. Prada Sánchez ${ }^{1}$

"Los administradores de carretas jamás serán constructores de locomotoras..."

J.A. Schumpeter
$\mathrm{E}^{\mathrm{l} \text { transporte es uno de los principales }}$ $\mathrm{E}_{\text {factores para la articulación del }}$ mercado nacional e internacional. Por lo tanto, "trasladar de un lugar a otro a personas o bienes" ha sido una

1 Maestro, Economista. Exdocente de la Facultad de Economía de la Universidad Nacional de San Antonio Abad del Cusco, Vice Rector de la Universidad Nacional Micaela Bastidas de Apurimac. Consultor Externo. 
preocupación tanto de gobernantes como de los productores y habitantes de toda sociedad a través de toda la historia de la humanidad.

A comienzos de 1802, la historia registra un hecho anecdótico que viene el caso recordarlo. Se reunieron en Londres los transportistas de carretas con el objeto de buscar soluciones a la congestión de carretas en todas las carreteras de Inglaterra. Recordemos, una carreta transportaba cuatro (4) pasajeros con un pequeño bulto, que era tirada por cuatro (4) caballos y dos conductores. El

"Desde mediados del siglo XIX hasta 1931 funcionaron 49 líneas férreas con $4,800 \mathrm{Km}$ de longitud. A 1992 quedaban cuatro, las que fueron privatizadas para incrementar $\mathrm{y}$ modernizarlas. Han pasado 25 años y funcionan dos."

propósito fue ampliar la carreta para transportar dos pasajeros más, para ello plantearon el fortalecimiento de las patas delanteras de los caballos, otros las patas traseras, otros el incremento del diámetro de las ruedas, otros sugirieron el incremento de los ejes, etc. Luego de dos meses de debate, concluyeron que cada quien desarrolle sus propuestas.

A fines de ese mismo año, el Ingeniero e inventor Inglés Richard Trevithck (13.04.1771 - 22.04.1833) dio a conocer la construcción de la primera locomotora del mundo, invento que lo vendió en 1803 a Samuel Hompay, quien a su vez en 1804 presentó el primer tren con capacidad de remolque de cinco vagones para transportar 30 personas por vagón. En 1830 se inauguró la primera línea férrea entre Liverpool y Manchester, una sola locomotora desplazó 37.5 carretas, 150 caballos y 75 cocheros. La articulación de este sistema de transporte facilitó el desarrollo de la industria, el comercio, finanzas y demás sectores que articularon el mercado nacional inglés y su ulterior conversión en país exportador de capitales en todo el mundo.

Este modelo fue aplicado inmediatamente por los demás países europeos, cuyo impacto fue un acelerado desarrollo de todos los sectores económicos, la historia lo registra como la I Revolución Industrial.

Nuestro país fue receptora de algunas ramas del desarrollo industrial, siendo uno de los primeros países en América Latina en crear la primera empresa naviera, la primera línea férrea y la primera línea aerocomercial. Esta vocación lamentablemente no ha continuado, por el contrario se nota el interés de "alguien" por desactivar y liquidar todas las modalidades de transporte masivo de carga y pasajeros en el Perú, llegando a tal situación que todas estas actividades se encuentran en manos de empresas extranjeras donde la participación de Chile es más que notoria.

El presente articulo describe el proceso de liquidación del transporte masivo de carga y pasajeros y formula propuestas para la recuperación de dichas actividades, con el propósito de obtener beneficios en este proceso de “competitividad y globalización".

\section{TRANSPORTE FERROVIARIO}

Es intención de este resumen histórico de los ferrocarriles en el Perú informar 
sobre los principales hechos que afectaron el desarrollo y la construcción de las líneas férreas en nuestro país. Basadre llama lamentable historia a la historia de los ferrocarriles, especialmente en el auge de su construcción o sea durante la segunda mitad del siglo diecinueve y la primera del veinte. Dice además al respecto:

"Los ferrocarriles en su mayor parte no llegaron a ser concluidos en los plazos estipulados y suscitaron complejos problemas alrededor de su financiación, o de su administración o de su terminación". No es casual que la mayor parte de los ferrocarriles ya no operen. De los pequeños hasta los más grandes, pasando por los quiméricos al Marañón, al Ucayali y Madre de Dios, tienen su historia y sus anécdotas. Desde que se iniciara la primera línea férrea en 1851 hasta que llegó a más de 4,800 kilómetros la red ferroviaria, sucedieron una serie de hechos a lo largo del

tiempo transcurrido desde que empezaron a correr. La historia de los ferrocarriles en el Perú está unida a la historia misma del país. En la guerra con Chile fueron destruidos más de 500 kilómetros de líneas, de un total de 1,500 que había en 1877. Se alcanzó otra vez dicha longitud recién en 1910.

\section{Hubo presidentes que se preocuparon}

mucho por dotar al Perú de un sistema

ferroviario acorde a las necesidades de desarrollo que el país requería. Castilla, fue el iniciador, luego Balta, los Pardo, Piérola y Leguía.

Desde mediados del siglo XIX hasta 1931 funcionaron 49 líneas férreas con $4,800 \mathrm{Km}$ de longitud. A 1992 quedaban cuatro, las que fueron privatizadas para incrementar y modernizarlas. Han pasado 25 años y funcionan dos líneas:

1. Callao - Lima - La Oroya - Cerro de Pasco $(535 \mathrm{Km}) \mathrm{y}$

2. Cusco - Machupicchu (112 Km)
3. Las otras: Cusco - Juliaca - Puno Arequipa, (940 Km); y Huancayo Huancavelica $(148 \mathrm{Km})$, se encuentran en abandono.

De los 4,800 Km que alguna vez tuvimos, hoy se encuentran operativas $654 \mathrm{Km}$ ese es el resultado de las privatizaciones. No incluimos el ferrocarril Tacna - Arica por estar en situación de abandono despiadado por disposición del Ministerio de Relaciones Exteriores.

\section{NO ES UN SUEÑO EL FERROCARRIL TUMBES - TACNA}

La construcción de esta vía férrea hoy es una necesidad urgente, pero para ello se requiere contar con gobernantes sin rodilleras. Su costo de doble vía a lo largo del litoral peruano $(3,079 \mathrm{Km})$, que incluye siete estaciones, 20 locomotoras y 500 vagones es aproximadamente US\$ $4.5 \mathrm{mil}$ millones.

El siguiente cuadro demuestra la alta rentabilidad de la ruta que proponemos.

Precios comparativos en el transporte masivo de carga por contenedor de $30 \mathrm{~T}$.

\begin{tabular}{llll}
\hline Transporte & Ruta & Contenedores & Precio Flete US\$ \\
\cline { 2 - 4 } $\begin{array}{l}\text { Camión } \\
\text { Arrastre }\end{array}$ & Lima - Piura & $34 \mathrm{~T}$ & $2,800.00$ \\
\hline Ferrocarril & Lima - Piura & $34 \mathrm{~T}$ (estimado) & 700.00 \\
\hline & & & \\
Cabotaje & Lima - Piura & $34 \mathrm{~T}$ & 500.00 \\
Marítimo & & & \\
\hline
\end{tabular}

Precios comparativos en el transporte masivo de pasajeros.

\begin{tabular}{llllll}
\hline Vía & Pasajess & $\begin{array}{l}\mathrm{N}^{\circ} \text { vagon es } \\
\text { / Bus }\end{array}$ & $\begin{array}{l}\text { Capacidd } \\
\text { Pax }\end{array}$ & $\begin{array}{l}\text { Pasaje } \\
\text { S/. }\end{array}$ & Total \\
\hline Ferrocarril & 60 & 25 & 1,500 & 30.00 & $45,000.00$ \\
\hline Bus & 45 & 33.3 & 1,500 & 120.00 & $180,000.00$ \\
\hline
\end{tabular}

El supuesto nos demuestra con claridad que para transportar 1,500 pasajeros de Lima a Piura se requieren 33.3 buses, cuyo precio promedio del pasaje (entre el precio del primer piso y el segundo) es de S/. 120.00. Si tomamos en cuenta el 
coeficiente internacional para calcular el precio del pasaje por persona por el mismo tramo por ferrocarril, este sería $1 / 6$, es decir S/.20.00, pero como estamos en el Perú, un precio tan barato no sería creíble, por lo que optamos por tomar en cuenta que sea $1 / 4$, es decir S/. 30.00, es obvio pensar en el tremendo impacto político que tendría en la población de menores recursos $(90 \%$ de la población nacional) la aplicación de esta política de transporte masivo generará la integración nacional y permitirá la acumulación de capitales tan necesarios para el desarrollo nacional. Un solo ferrocarril con 25 vagones reemplazará a 33.3 buses con el consiguiente ahorro en combustible contaminante, además los accidentes en carreteras originado mayormente por los buses y camiones-tracto disminuirán a niveles mínimos.

Los accidentes en las carreteras y pistas de nuestro país son cada vez más frecuentes y se vienen convirtiendo en un problema nacional, que no quieren aceptarlo los funcionarios del Ministerio de Transportes y Comunicaciones no quiere decir que no lo sea. El problema es que todos los ministros de esa cartera desde el año 1980, es decir desde hace 35 años no tienen una sola idea para convertirla en política nacional de transporte. No tienen un diseño de una Política de Transporte Masivo, se vienen adecuando a la importación de vehículos, que en estos 35 años ha saturado no solo nuestra principal vía de articulación: la carretera asfaltada Panamericana Norte y Sur, sino todas las vías transversales.

El siguiente cuadro nos muestra con claridad, la presencia aplastante de los camiones de arrastre-carga nacional, pistas y carreteras, el problema se viene convirtiendo en nacional.

Flota Nacional de Transporte Terrestre de Carga y Pasajeros

\begin{tabular}{ll}
\hline Buses interprovinciales de pasajeros & 8,465 \\
\hline Camiones de arrastre (carga) & 199,354 \\
Vehículos Matpel & 11,183 \\
\hline Camiones para transporte de carga internacional & 18,014 \\
\hline Buses Internacionales para transporte de personas & 564 \\
\hline Servicio de transporte sin autorización (Informales) & s/d \\
\hline & 237,580 \\
\hline
\end{tabular}

Informe de la Sra. Elvira Moscoso Cabrera, Superintendente de la Superintendencia de Transporte Terrestre, Personal, Carga y Mercancias - SUTRAN, ante la Comisión de Transportes y Comunicaciones del Congreso de la República. 9 de Abril de 2013.

Finalmente, todas las empresas de transporte terrestre interprovincial de buses utilizan un tiempo de desplazamiento entre 14 y 16 horas en la ruta Lima - Piura $(992 \mathrm{Km})$, a una velocidad promedio de $70 \mathrm{Km} / \mathrm{h}$. El transporte por ferrocarril se desplazaría por esa misma ruta en 9 horas.

El tramo de la ruta Lima - Arequipa es aproximadamente $1,000 \mathrm{Km}$, siendo el tiempo de desplazamiento un promedio de 16 horas, debido a la congestión hasta Nasca y la trocha angosta de Nasca Arequipa. El transporte por ferrocarril disminuirá a tan solo 10 horas.

El ferrocarril Tumbes - Tacna es pues un sueño que urge materializarlo. ¿será necesario incluir en la agenda de nuestros reclamos, para que la insensible burocracia limeña que anida en el sector transporte tome conciencia...?matpel carga internacional; sumados a los buses de transporte de pasajeros nacionales y extranjeros suman 237,580 , según información de 2013, referencia que al día de hoy pasa los 260,000 vehículos. Esta es una las causas principales que generan accidentes de tránsito, que sumado a las trochas angostas de nuestras

\section{REFERENCIAS}

-1. BASADRE Grohmann, Jorge (1970). Historia de la República. Lima.

-2. Informe de la Superintendente de la SUTRAN ante la Comisión de Transportes y Comunicaciones del Congreso de la República. 9 de abril de 2013.

-3. OMS (2012), Informe se la Situación Mundial de la Seguridad Vial. Ginebra - Suiza. 